\title{
Die schweizerischen Gemeinden und das Sechseckmodell
}

Rudolf Koblet

In seinem Bericht vom «Symposium international de géographie de l'habitat et des paysages agraires d'Europe» in Liège erwähnte W. A. Gallusser in der "Geographica Helvetica» 1969/4, S. 212, eine Arbeit von A. R. H. Baker über die sogenannten Kontaktzahlen von 300 französischen Landgemeinden. Da der Verfasser des vorliegenden Artikels in seiner 1966 unter Prof. Dr. H. Gutersohn an der ETH ausgeführten Diplomarbeit - «Beziehungen zwischen natürlichen und politischen Grenzen an Schweizer Beispielen" - ähnliche Probleme untersuchte, seien die wichtigsten Ergebnisse im folgenden kurz dargelegt.

Anteil der Gemeinden

mit $K=6$ bzw. $6 \pm 1$
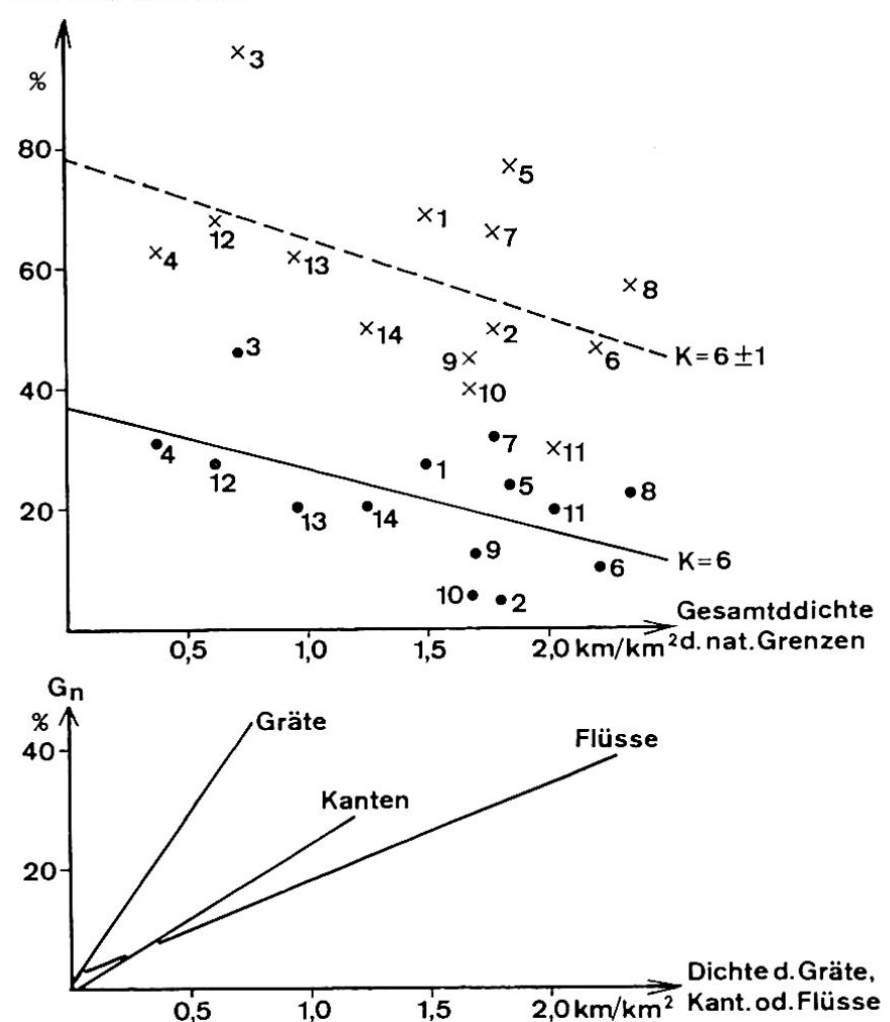

Figur 1. Oben: Der Zusammenhang zwischen der Kontaktzahl $\mathrm{K}=6(6 \pm 1)$ und der Dichte der natürlichen Grenzen

Unten: Die Anteile der natürlichen Grenzen an den Gemeindegrenzen $\left(G_{n}\right)$
Die Grenzen der heutigen Gemeinden scheinen sehr oft aus Zusammenwachsgrenzen von Rodungsinseln entstanden zu sein. Unter der Annahme, daß diese einigermaßen regelmäßig verteilt sind, sollten die Gemeindegrenzen theoretisch ein Sechsecknetz bilden, das heißt jede Gemeinde würde an sechs andere grenzen, oder ihre Kontaktzahl $\mathrm{K}$ wäre 6 . Praktisch ist dieses Netz jedoch durch die Topographie deformiert, vor allem Bäche, Flüsse und Grate werden als natürliche, gut erkennbare Leitlinien für die politischen Grenzen bevorzugt; Landabtausch und Eingemeindungen tragen das ihre $\mathrm{zu}$ einer Verschleierung früherer Zustände bei.

Vor großer Wichtigkeit ist die Kenntnis der Häufigkeiten, mit der die einzelnen Werte der Kontaktzahlen auftreten, und deren räumliche Verteilung. Deshalb wurde aufgrund der Tafel 2 des Atlasses der Schweiz eine Karte der Kontaktzahlen erstellt, die die ganze Schweiz umfaßt. Dabei wurden Enklaven wie ganze Gemeinden behandelt, während bei Gemeinden, die an einen See grenzen, letzterer ebenfalls als nächste "Nachbargemeinde» galt. Die Karte zeigt ein großes Durcheinander der Kontaktzahlen über das ganze Gebiet. Der Wert $\mathrm{K}=6$ is $\hat{\imath}$ nicht dominierend (vgl. Tabelle 1), scheint aber im Mittelland häufiger zu sein als in den Alpen, was eine quantitative Abhängigkeit von der Dichte der natürlichen Grenzen vermuten läßt.

Die durchschnittliche Kontaktzahl beträgt 5,73.

Tabelle 1 Die Häufigkeiten der Kontaktzahlen

$\begin{array}{ccc}\begin{array}{c}\text { Kontaktzahl } \\ \text { K }\end{array} & \begin{array}{c}\text { Anzahl der } \\ \text { Gemeinden }\end{array} & \begin{array}{c}\text { Anteil in } \\ \%\end{array} \\ 1 & 5 & 0,1 \\ 2 & 40 & 1,3 \\ 3 & 197 & 6,2 \\ 4 & 594 & 18,8 \\ 5 & 734 & 23,4 \\ 6 & 703 & 22,4 \\ 7 & 404 & 12,8 \\ 8 & 225 & 7,1 \\ 9 & 128 & 4,1 \\ 10 & 71 & 2,2 \\ 11-17 & 52 & 1,6\end{array}$


Ebenso wie bei Baker2 ist das Sechseckmodell nicht vorherrschend. Mögliche Gründe, die ein ehemals vielleicht vorhanden gewesenes "Sechseck» verändert haben können, wurden bereits erwähnt: natürliche Grenzen und Landabtausch. Vielleicht ist die Tatsache, daß 58,6\% der Gemeinden die Kontaktzahlen $\mathrm{K}=6 \pm 1$ aufweisen, ein Hinweis, daß die «Sechsecktheorie» doch nicht ganz aus der Luft gegriffen ist. Die folgende Untersuchung über den Einfluß der natürlichen Grenzen auf die Häufigkeit der Gemeinden mit den Kontaktzahlen $\mathrm{K}=6$ oder $\mathrm{K}=6 \pm 1$ wird diese Vermutung bestätigen.

Für eine Stichprobe von 14 Quadraten mit $10 \mathrm{~km}$ Seitenlänge (Lage und Koordinaten siehe Tabelle 2) wurde zuerst die quantitative Beziehung zwischen der Dichte der natürlichen Grenzen (Grate, Kanten und Flüsse) und dem prozentualen Anteil dieser Formen von natürlichen Grenzen an den Gemeindegrenzen ermittelt. Als Grat wurde dabei eine Wasserscheide beliebiger Ordnung bezeichnet, die in der Landeskarte 1:50000 durch ein scharfes Umbiegen der Höhenkurven dargestellt ist. Eine Kante ist ein markanter Gefällswechsel, bei dem die größere Neigung unten ist. Unter einem Flu $\beta$ wird jedes in der erwähnten Karte dargestellte Fließgewässer verstanden. In der Abbildung sind unten die Regressionsgeraden der genanten Beziehungen eingezeichnet. Es treten folgende Korrelationen auf:

Tabelle 2 Lage und Koordinaten der Quadratmittelpunkte. (Die Nummern entsprechen denen in der Abbildung)

Lage
1. Gelterkinden
2. Märstetten
3. Wohlen
4. St-Imier
5. Lumnezia
6. Lugano
7. Sottens
8. Langnau
9. P. Molare
10. Säntis
11. Sierre
12. Genf
13. Avenches
12. Münsingen

Koordinaten

$\begin{array}{ll}\text { BL } & 635 / 259 \\ \text { TG } & 719 / 273 \\ \text { AG } & 665 / 247 \\ \text { BE } & 567 / 223 \\ \text { GR } & 732 / 175 \\ \text { TI } & 715 / 103 \\ \text { VD } & 545 / 163 \\ \text { BE } & 625 / 193 \\ \text { TI } & 710 / 148 \\ \text { AR/SG } & 747 / 237 \\ \text { VS } & 603 / 125 \\ \text { GE } & 495 / 117 \\ \text { FR/VD } & 573 / 189 \\ \text { BE } & 609 / 189\end{array}$

$\begin{array}{lcc} & \text { r } & \text { P } \\ \text { Grate } & 0,93 & 0,001 \\ \text { Kanten } & 0,91 & 0,001 \\ \text { Flüsse } & 0,62 & 0,05\end{array}$

Damit ist für diese Stichprobe erwiesen, daß die natürlichen Grenzen um so eher als Leitlinien für politische Grenzen benützt werden, je größer die Dichte der ersteren ist.

Innerhalb der Quadrate und in ihrer näheren Umgebung wurde nun die Häufigkeit der Gemeinden mit den Kontaktzahlen $\mathrm{K}=6$ oder $\mathrm{K}=6 \pm 1$ ermittelt und in Abhängigkeit der Gesamtdichte von Graten, Kanten und Flüssen der zugehörigen Quadrate dargestellt (siehe Abbildung). Die Regressionsgeraden zeigen deutlich, daß der Anteil der Gemeinden mit $\mathrm{K}=6$ oder $6 \pm 1$ mit steigender Dichte der natürlichen Grenzen abnimmt. Die Korrelationskoeffizienten betragen 0,55 oder 0,50, beide gesichert für $P=0,05$. Die «störenden» Einflüsse der natürlichen Grenzen auf das theoretisch geforderte Sechsecknetz sind damit zumindest für die vorliegende Stichprobe quantitativ nachgewiesen.

\section{Zusammenfassung}

Es wird festgestellt, daß das «Sechseckmodell», wie es bei zusammenwachsenden Rodungsinseln zu erwarten wäre, bei den schweizerischen Gemeinden nicht dominierend ist. Natürliche Grenzen, Landabtausch usw. deformieren das theoretisch geforderte Grenzliniennetz. An einer Stichprobe wird quantitativ nachgewiesen, daß der Anteil der Gemeinden mit sechs nächsten Nachbargemeinden um so mehr sinkt, je größer die Dichte der natürlichen Grenzen ist.

\section{Quellen}

1. Atlas der Schweiz. Wabern-Bern 1965 ff.

2. Gallusser, W.A.: Siedlungsgeographie und Agrarlandschaften Europas. Geogr. Helv. 1969/4, S. 210-13.

3. Haggett, P.: Locational Analysis in Human Geography. London 1965.

4. Koblet, R.: Beziehungen zwischen natürlichen und politischen Grenzen an Schweizer Beispielen. 1966 (ungedruckt).

5. Landeskarte der Schweiz 1:50 000. WabernBern. 